\title{
A NOTE ON THE PRODUCT OF $\mathscr{F}$-SUBGROUPS IN A FINITE GROUP
}

\author{
by ANGEL CAROCCA
}

(Received 3rd March 1994)

Saturated formations are closed under the product of subgroups which are connected by certain permutability properties.

1991 Mathematics subject classification: 20F17, 20D40

All groups we consider are finite. It is well known that the product of supersolvable normal subgroups is not supersolvable in general (see Huppert [3]).

In [1], Asaad and Shaalan proved the following result:

Let $G=G_{1} G_{2}$ be a group such that $G_{1}$ and $G_{2}$ are supersolvable subgroups. If every subgroup of $G_{1}$ is permutable with every subgroup of $G_{2}$, then $G$ is supersolvable.

If $G_{1}$ and $G_{2}$ are subgroups of a group $G$ such that every subgroup of $G_{1}$ is permutable with every subgroup of $G_{2}$, we say that $G_{1}$ and $G_{2}$ are totally permutable.

In [6], Maier proved that Asaad and Shaalan's result is a special case of a general completeness property of all saturated formations which contain the class of supersolvable groups. In [6], the following theorem is proved:

Let $G=G_{1} G_{2}$ be a group such that $G_{1}$ and $G_{2}$ are totally permutable subgroups. Let $\mathscr{F}$ be a saturated formation which contains the class of supersolvable groups. If $G_{1}$ and $G_{2}$ lie in $\mathscr{F}$, then so does $G$.

In this paper we give a generalization for an arbitrary number of factors of Maier's result. We prove:

Theorem 1. Let $G=G_{1} G_{2} \ldots G_{r}$ be a group such that $G_{1}, G_{2}, \ldots, G_{r}$ are pairwise totally permutable subgroups of $G$. Let $\mathscr{F}$ be a saturated formation which contains the class of supersolvable group. If for all $i \in\{1,2, \ldots, r\}$ the subgroups $G_{i}$ are in $\mathscr{F}$, then $G \in \mathscr{F}$.

If $G_{1}$ and $G_{2}$ are totally permutable subgroups of a group $G$, then $\langle x, y\rangle=\langle x\rangle\langle y\rangle=$ $\langle y\rangle\langle x\rangle$ is a supersolvable subgroup, for each $x \in G_{1}$ and $y \in G_{2}$, by $([4$, p. 722 , Th. 10.1]). If $G_{1}$ and $G_{2}$ are subgroups of a group $G$ and $\mathscr{L}$ is a non-empty class of groups, 
we say that $G_{1}$ and $G_{2}$ are $\mathscr{L}$-connected, whenever for each $x \in G_{1}$ and $y \in G_{2}$ we have $\langle x, y\rangle \in \mathscr{L}$.

Assuming this definition, we prove the following:

Theorem 2. Let $G=G_{1} G_{2}, \ldots G_{r}$ be a group such that $G_{1}, G_{2}, \ldots, G_{r}$ are pairwise permutable subgroups of $G$. Let $\mathscr{L}=\mathscr{N}$ be the class of nilpotent groups and let $\mathscr{F}$ be a saturated formation such that $\mathscr{N} \subseteq \mathscr{F}$. If for every pair $i, j \in\{1,2, \ldots, r\}, i \neq j$, the subgroups $G_{i}$ and $G_{j}$ are $\mathcal{N}$-connected $\mathscr{F}$-groups, then $G \in \mathscr{F}$.

This paper follows part of the author's Doctoral Thesis written under supervision of Professor R. Maier. The author also thanks the Brazilian Research Council (CNPq) for the scholarship granted.

\section{Proofs of our Theorems}

To prove theorem 1, we first generalize Lemma 2 of [6]:

Lemma 1. Let the group $G=G_{1} G_{2} \ldots G$, be the product of the pairwise totally permutable subgroups $G_{1}, G_{2}, \ldots, G_{r}$ of $G$.

(a) If $|G|>1$, then there exists $i \in\{1,2, \ldots, r\}$ such that $G_{i}$ contains a nonidentity normal subgroup of $G$.

(b) For every pair $i, j \in\{1,2, \ldots, r\}, i \neq j$, we have that $G_{i} \cap G_{j} \leqq \mathrm{~F}\left(G_{i} G_{j}\right)$, where $\mathrm{F}\left(G_{i} G_{j}\right)$ denotes the Fitting subgroup of $G_{i} G_{j}$.

Proof. (a) Let $p$ denote the largest prime divisor of $|G|$. Certainly $p$ divides at least one of $\left|G_{1}\right|,\left|G_{2}\right|, \ldots,\left|G_{r}\right|$. Let $x$ be a $p$-element of the union set $G_{1} \cup G_{2} \cup \ldots \cup G_{r}$ of maximal order and suppose $x \in G_{1}$. Let $R$ be the subgroup of order $p$ in $\langle x\rangle$. As in the proof of Lemma 2 in [6], we conclude that $G_{i}$ normalizes $R$ for all $i \in\{2, \ldots, r\}$. Therefore the normal closure

$$
R^{G}=R^{G_{2} G_{3} \ldots G_{r} G_{1}}=R^{G_{1}} \leqq G_{1}
$$

is a nonidentity normal subgroup of $G$ contained in $G_{1}$.

(b) This is (b) of Lemma 2 in [6].

Theorem 1. Let $G=G_{1} G_{2} \ldots G_{r}$ be a group such that $G_{1}, G_{2}, \ldots, G_{r}$ are pairwise totally permutable subgroups of $G$. Let $\mathscr{F}$ be a saturated formation which contains the class of supersolvable groups. If for all $i \in\{1,2, \ldots, r\}$ the subgroups $G_{i}$ are in $\mathscr{F}$, then $G \in \mathscr{F}$.

Proof. Suppose the theorem is false and let $G$ be a counterexample of smallest order with $r$ least possible. Then $1<G_{i}<G$ for all $i \in\{1,2, \ldots, r\}$. We will prove a series of 
items under this assumption. They will lead us to a contradiction. Certainly the hypothesis is inherited by factor groups.

(i) The group $G$ has a unique minimal normal subgroup $N$ and the Frattini subgroup $\Phi(G)=1$.

Since the hypothesis is inherited by quotient groups, by the minimality of $|G|$, every proper quotient group $G / M(1 \neq M \unlhd G)$ lies in $\mathscr{F}$. Since $\mathscr{F}$ is a saturated formation, the minimal normal subgroup $N$ of $G$ is unique and $\Phi(G)=1$.

(ii) There exists $i \in\{1,2, \ldots, r\}$, such that $N \leqq G_{i}$.

This is Lemma 1 (a).

We suppose $N \leqq G_{1}$.

(iii) $N$ is an elementary abelian p-group, for some prime number $p$.

Otherwise $N$ is the direct product of nonabelian simple groups. Because of the uniqueness of $N$ we have $C_{G}(N)=1$, the centralizer of $N$ in $G$. Put $H=G_{1} G_{2}$. By Lemma 1 (b), we have that $G_{1} \cap G_{2} \leqq \mathbf{F}(H)$. So, $N \cap G_{2}=N \cap G_{1} \cap G_{2} \leqq N \cap \mathbf{F}(H) \leqq \mathbf{F}(N)=1$.

Let $X \leqq N$. Since $G_{1}$ and $G_{2}$ are totally permutable, we have that $G_{2} X=X G_{2}$. So $X=X\left(N \cap G_{2}\right)=N \cap X G_{2} \unlhd G_{2} X$. Hence, $G_{2}$ normalizes every subgroup of $N$. By ([2, Th. 2.2.1.]), the commutator group $\left[G_{2}, N\right]$ is in the centre of $N$. Therefore, $G_{2} \leqq$ $\mathrm{C}_{G}(N)=1$, a contradiction.

(iv) There is a complement $V$ of $N$ in $G,|N|>p$ and $\mathbf{C}_{G}(N)=N=\mathbf{F}(G)$.

This is shown in the same way as the items (iv)/(v) in [6].

It is clear that, if $N \leqq G_{i}$, then $U_{i}=G_{i} \cap V$ is a complement of $N$ in $G_{i}$.

(v) There exists $i \in\{2,3, \ldots, r\}$, such that $N \$ G_{i}$.

Suppose $N \leqq \bigcap_{i=1}^{r} G_{i}$. Then $U_{i}=G_{i} \cap V$ is a complement of $N$ in $G_{i}$. Let $X \leqq N$. Since $r>1$, we have $X U_{i}=U_{i} X$ and $X=X\left(U_{i} \cap N\right)=N \cap U_{i} X \unlhd X U_{i}$. Since $N$ is abelian, we have $X \unlhd G_{i}$ for all $i \in\{1,2, \ldots, r\}$. Therefore, by the minimality of $N$, we conclude $|N|=p$, against (iv).

We renumber the indices in such a way that $N \leqq G_{i}$ for all $i \in\{1,2, \ldots, s\}$ and $N \leqq G_{j}$ for all $j \in\{s+1, \ldots, r\}$. Let $K=G_{s+1} G_{s+2} \ldots G_{r}$.

(vi) For all $j \in\{s+1, \ldots, r\}$ we have $N \cap G_{j}=1$.

Let $j \in\{s+1, \ldots, r\}$ and $D=N \cap G_{j}$. Suppose $D>1$. Let $i \in\{1,2, \ldots, s\}$. We have that $N \leqq G_{i}$ and $U_{i}=V \cap G_{i}$ is a complement of $N$ in $G_{i}$. Since $D \leqq G_{j}$ and $i \neq j$, we have $D U_{i}=U_{i} D$. Hence $D \unlhd U_{i} D$. Therefore $D \unlhd G_{i}$ for all $i \in\{1,2, \ldots, s\}$. It follows that $N=D^{G}=D^{G_{s+1} \ldots G_{r}} \leqq G_{s+1} \ldots G_{r}=K$.

Since $K$ is the product of pairwise totally permutable subgroups, once more by Lemma 1 (a) there exists $1 \neq L \subseteq K$ such that, for example $L \leqq G_{s+1}$.

Consider $J=N \cap L \subseteq K$. Since $J \leqq G_{s+1}$ we have that the subgroups $G_{i}$ normalize $J$, for all $i \in\{1,2, \ldots, s\}$. Hence $J \leq G$. By the minimality of $N$ we conclude $J=N$ or $J=1$. Since $N \leq G_{s+1}$, we have $J=1$. Therefore, the commutator $[N, L] \leqq L \cap N=J=1$. So $L \leqq \mathrm{C}_{G}(N)=N$. Since $L \leqq G_{s+1}$, we have that $G_{i}$ normalizes $L$, for all $i \in\{1,2, \ldots, s\}$. Hence, $L=N \leqq G_{s+1}$, a contradiction.

(vii) For all $j \in\{s+1, \ldots, r\}$, we have that $G_{j}$ normalizes every subgroup of $N$.

If $X \leqq N$, then $X G_{j}=G_{j} X$ and $X=X\left(G_{j} \cap N\right)=G_{j} X \cap N \unlhd G_{j} X$ by (vi).

(viii) We have $s=1$. 
Suppose $N \leqq G_{i}$ and $i>1$. Let $X \leqq N$ and $U_{1}=G_{1} \cap V$. We have that $U_{1} X=X U_{1}$, (because $i>1$ ). So, $G_{1}$ normalizes $X$. Similarly $G_{i}$ normalizes $X$, for all $G_{i}$ such that $N \leqq G_{i}$. Hence $X \unlhd G$, by (vii). So, $|N|=p$, against (ii).

By (vii) and (viii) we have that $N$ is a minimal normal subgroup of $G_{1}$.

(ix) For all $j \in\{2, \ldots, r\}$ we have $G_{1} \cap G_{j}=1$.

Let $D=G_{1} \cap G_{j}$. By Lemma $2(\mathrm{~b})$, we have $D \leqq \mathrm{~F}\left(G_{1} G_{j}\right)$. Since $N$ is minimal normal in $G_{1}$, ([4], p. 277 , Th. $\left.4.2(\mathrm{e})\right)$, we have $\mathrm{F}\left(G_{1}\right) \leqq \mathrm{C}_{G}(N)=N$. It follows that $D \leqq N \cap G_{j}=$ 1 , by (vi).

By (vi) and (vii), we have that for all $j \in\{2, \ldots, r\}$ the subgroups $G_{j}$ are faithfully represented on the vector space $N$ by scalar transformations. Let $U_{1}^{x}$ be a conjugate of $U_{1}=G_{1} \cap V$ in $G_{1}$.

(x) For all $j \in\{2, \ldots, r\}$ we have that $G_{j}$ centralizes $U_{1}^{x}$.

Clearly, $G_{j} U_{1}^{x}=U_{1}^{x} G_{j}$ and $U_{1}^{x} G_{j} \cap N=U_{1}^{x} G_{j} \cap G_{1} \cap N=U_{1}^{x}\left(G_{j} \cap G_{1}\right) \cap N=U_{1}^{x} \cap N=$ 1. So, $U_{1}^{x} G_{j}$ is faithfully represented on $N$. Since $G_{j}$ is represented by scalar transformations, we have that $G_{j}$ centralizes $U_{1}^{x}$.

(xi) The contradiction

Clearly, $N \neq G_{1}$, so $U_{1}$ is a non-normal subgroup of $G_{1}$. It follows that $G_{1}=$ $\left\langle U_{1}^{x} / x \in G_{1}\right\rangle$. Hence, for all $j \in\{2, \ldots, r\}, G_{j}$ centralizes $G_{1}$, by $(x)$. Therefore $G_{j} \leqq \mathbf{C}_{G}(N)=$ $N$ and $G_{j}=1$, by (vi).

Theorem 2. Let $G=G_{1} G_{2} \ldots G_{r}$ be a group such that $G_{1}, G_{2}, \ldots, G_{r}$ are pairwise permutable subgroups of $G$. Let $\mathscr{L}=\mathscr{N}$ be the class of nilpotent groups and let $\mathscr{F}$ be a saturated formation such that $\mathscr{N} \subseteq \mathscr{F}$. If for every pair $i, j \in\{1,2, \ldots, r\}, i \neq j$, the subgroups $G_{i}$ and $G_{j}$ are $\mathscr{N}$-connected $\mathscr{F}$-groups, then $G \in \mathscr{F}$.

Proof. Suppose the theorem is false and let $G$ be a counterexample of smallest order. Since the hypothesis is inherited by quotients, we conclude that $G$ has a unique minimal normal subgroup $N$. Since $\mathscr{F}$ is saturated, we have $\Phi(G)=1$.

Let $p$ be a prime number and $i, j \in\{1,2, \ldots, r\}$, such that $i \neq j$. Let $x \in G_{i}$ be a $p$-element and $y \in G_{j}$ a $p^{\prime}$-element. Since $\langle x, y\rangle$ is nilpotent, we have that $y$ centralizes $x$. Let $P_{i} \in S y l_{p}\left(G_{i}\right)$. Since $\mathbf{O}^{p}\left(G_{j}\right)$ is generated by all $p^{\prime}$-elements of $G_{j}$, we have $\mathbf{O}^{p}\left(G_{j}\right) \leqq \mathbf{C}_{G}\left(P_{i}\right)$. For the definition of $\mathbf{O}^{p}\left(G_{j}\right)$ see $([7$, p. 142]).

Set $G_{j}^{*}=\bigcap_{p} \mathbf{O}^{p}\left(G_{j}\right)$. The above consideration implies that $G_{i} \leqq \mathbf{C}_{G}\left(G_{j}^{*}\right)$. Since our argument is true for all $i \in\{1,2, \ldots r\}$, such that $i \neq j$, we have that $G_{j}^{*} \unlhd G$.

(I) Suppose $G_{j}^{*} \neq 1$, for some $j \in\{1,2, \ldots, r\}$.

Because of the uniqueness of $N$ we have $N \leqq G_{j}^{*}$.

(a) If $N$ is solvable, then $N=\mathrm{C}_{G}(N)$ and $G_{i} \leqq N \leqq G_{j}^{*}$, for all $i \in\{1,2, \ldots, r\}$, with $i \neq j$. It follows that $G=G_{j} \in \mathscr{F}$.

(b) If $N$ is not solvable, then $\mathbf{C}_{G}(N)=G_{i}=1$ for all $i \in\{1,2, \ldots, r\}$ with $i \neq j$. Again we have $G=G_{j} \in \mathscr{F}$.

(II) Suppose $G_{j}^{*}=1$ for all $j \in\{1,2, \ldots, r\}$. Now $G_{j}$ is nilpotent for all $j \in\{1,2, \ldots, r\}$. Hence, $G_{j}=P_{j} \times \mathbf{O}^{p}\left(G_{j}\right)$, for every prime number $p$.

Let $i, j \in\{1,2, \ldots, r\}$ such that $i \neq j$. By $([4$, p. 676, Th. 4.7]) we have that $P_{i} P_{j} \in S y l_{p}\left(G_{i} G_{j}\right)$. Hence $P_{1} P_{2} \ldots P_{r} \in S y l_{p}(G)$. Since for all $i \in\{1,2, \ldots, r\}$ we have 
$\mathbf{O}^{p}\left(G_{i}\right) \leqq \mathrm{C}_{G}\left(P_{1} P_{2} \ldots P_{r}\right)$, we conclude that $G_{i} \leqq \mathbf{N}_{G}\left(P_{1} P_{2} \ldots P_{r}\right)$ and therefore $P_{1} P_{2} \ldots P_{r} \unlhd G$. It follows that $G \in \mathscr{N} \subseteq \mathscr{F}$.

The following example shows that Theorem 2 is not true when $\mathscr{N} \varsubsetneqq \mathscr{L} \subseteq \mathscr{F}$, without additional hypothesis (see also the Example given in [6]):

Example. Let $G=S_{4}$ be the symmetric group of degree 4. Let $G_{1}$ be the normal subgroup of order 4 of $G$ and let $G_{2}$ be a subgroup of order 6 of $G$. Clearly, $G=G_{1} G_{2}$. Let $\mathscr{L}=\mathscr{N} \mathscr{A}=\mathscr{F}$ be the class of all groups whose commutator subgroups are nilpotent. Clearly, $G_{1}$ and $G_{2}$ are $\mathscr{N} \mathscr{A}$-connected $\mathscr{F}$-groups, but $G \notin \mathscr{F}$.

In view of the fact that the finite simple groups are 2-generated, the following seems to be reasonable:

Conjecture. Let $\mathscr{S}$ be the class of solvable groups. If the group $G=G_{1} G_{2} \ldots G_{r}$ is the

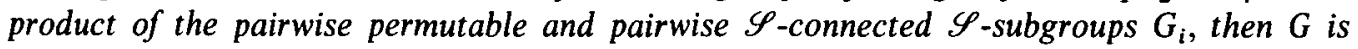
solvable.

To mention the solution of a particular case of this conjecture, we introduce the following notation: Let $\mathscr{T}$ be the class of groups having Sylow-tower for the prime numbers arranged in decreasing order.

Proposition. Let $G=G_{1} G_{2} \ldots G_{r}$ be a group such that $G_{1}, G_{2}, \ldots G_{r}$ are pairwise permutable and pairwise $\mathscr{T}$-connected supersolvable subgroups of $G$. Then $G$ is a $\mathscr{T}$-group. In particular, $G$ is solvable.

Proof. Suppose the proposition is false. Let $G$ be a counterexample of smallest order with $r$ least possible. Every quotient group of $G$ satisfies the hypothesis of the proposition. Because of the minimality of $|G|$, every proper quotient group is a $\mathscr{T}$-group.

Let $p$ denote the largest prime number divisor of $|G|$. We may assume that $p$ divides $\left|G_{1}\right|$. We have to produce a nonidentity normal $p$-subgroup $N$ of $G$.

Because of the supersolvability of $G_{1}$, we can choose $\langle x\rangle$ a normal subgroup of $G_{1}$, with $|\langle x\rangle|=p$. We show $\langle x\rangle$ is subnormal in $G$. Then $N=\langle x\rangle^{G}$ is a normal $p$-subgroup of $G$.

First we show that $r \leqq 2$. If $r \geqq 3$, then $H=G_{1} G_{2} \ldots G_{r-1}$ and $K=G_{1} G_{2} \ldots G_{r-2} G_{r}$ are $\mathscr{T}$-groups, since $r$ is least possible. Hence $\langle x\rangle$ is subnormal in $H$ and $K$. By $([5$, p. 239, Th. 7.7.1]) we have that $\langle x\rangle$ is subnormal in $H K=G$. So $G=G_{1} G_{2}$.

Let $g \in G$. Write $g=g_{1} g_{2}$ with $g_{1} \in G_{1}$ and $g_{2} \in G_{2}$. Since $\langle x\rangle \unlhd G_{1}$, we have that $x^{g_{1}}=x^{i}$ with $1 \leqq i \leqq p$. By hypothesis $\left\langle x, g_{2}\right\rangle$ is a $\mathscr{T}$-group, thus $\left\langle x, g_{2}\right\rangle_{p} \leq\left\langle x, g_{2}\right\rangle$, where $\left\langle x, g_{2}\right\rangle_{p}$ denotes the Sylow-p-subgroup of $\left\langle x, g_{2}\right\rangle$. Therefore $x, x^{g_{2}} \in\left\langle x, g_{2}\right\rangle_{p}$ and $x^{g}=$ $x^{g_{1} g_{2}}=\left(x^{i}\right)^{g_{2}}=\left(x^{g_{2}}\right)^{i} \in\left\langle x, g_{2}\right\rangle_{p}$. It follows that $\left\langle x, x^{g}\right\rangle$ is a $p$-group, for all $g \in G$. By $([7, \mathrm{p}$. 195, Th. 4.8]) we have that $\langle x\rangle$ is subnormal in $G$. 


\section{REFERENCES}

1. M. AsaAd and A. ShaAlan, On the supersolvability of finite groups, Arch. Math. 53 (1989), 318-326.

2. C. COOPER, Power automorphisms of a group, Math. Z. 107 (1968), 335-356.

3. B. HUPPERT, Monomiale Darstellungen endlicher Gruppen, Nagoya Math. J. 6 (1953), 93-94.

4. B. HUPPERT, Endliche Gruppen I (Springer-Verlag, Berlin/Heidelberg/New York, 1967).

5. J. Lennox and St. E. Stonehewer, Subnormal Subgroups of Groups (Clarendon Press, Oxford, 1987).

6. R. MAIER, A completeness property of certain formations, Bull. London Math. Soc. 24 (1992), 540-544.

7. M. Suzuki, Group Theory I (Springer-Verlag, Berlin/Heidelberg/New York, 1982).

Departamento de Matemáticas

Universidad Técnica Federico Santa Maria

Casilla 110-V, Valparaíso

ChILE

Current address

Facultad de Matemáticas

Pontificia Universidad Católica de Chile

Casilla 306

SANTIAGO 22

CHILE

E-mail address: ACAROCCA@MAT.PUC.CL 